\title{
Gaps in neonatal thermal care in low-resource settings: A web- based survey of healthcare workers.
}

Michiko Kyokan ( $\square$ Michiko.Kyokan@etu.unige.ch )

University of Geneva Medical Centre: Universite de Geneve Faculte de Medecine https://orcid.org/0000-0001-5539-1828

Veena Jirapaet

Chulalongkorn University Faculty of Nursing

Flavia Rosa-Mangeret

University of Geneva Faculty of Medicine: Universite de Geneve Faculte de Medecine

Giorgia Brambilla Pisoni

Federal Polytechnic School of Lausanne: Ecole Polytechnique Federale de Lausanne

Riccardo E Pfister

University of Geneva Faculty of Medicine: Universite de Geneve Faculte de Medecine

\section{Research}

Keywords: neonate, thermal care, hypothermia, cold stress, low-resource settings

Posted Date: October 21st, 2021

DOI: https://doi.org/10.21203/rs.3.rs-980430/v1

License: (a) (i) This work is licensed under a Creative Commons Attribution 4.0 International License. Read Full License 


\section{Abstract \\ Objective}

To explore the gaps in knowledge and practice in neonatal thermal care among healthcare workers in low-resource settings.

\section{Methods}

We conducted a 2-round, web-based survey of a purposive and snowball sample of healthcare workers in neonatal care in low-resource settings globally. The questionnaire was developed using themes of neonatal thermal care extracted from existing neonatal care guidelines, including WHO's. The survey asked multiple-choice questions, supplemented by open-ended questions to capture first hand insights and information on neonatal thermal care. Results of the survey were analysed using Microsoft Excel. Data was collated and summarized using descriptive measures.

\section{Results}

Almost all participants acknowledged the importance of all the WHO warm chain elements, however, fewer participants responded positively regarding the practice of this warm chain. Only $56 \%$ of the participants acknowledged the usefulness of checking the peripheral temperature by hand-touch. The usefulness of the core temperature was valued higher than that of the peripheral temperature as an indicator of cold stress, with $70 \%$ and $58 \%$ agreement, respectively. Opinions diverged regarding the peripheral temperatures, including apparent inaccuracy compared to rectal or axillary temperature. Preferences on rewarming strategies widely differed among participants and so did the availability of warming equipment in their institutions.

\section{Conclusions}

We identified the general acknowledgment of the importance of the WHO warm chain but also its limited practice. We also identified that an inadequate understanding of cold stress underestimates the potential benefits of peripheral temperatures and leads to missed opportunities for timely prevention of hypothermia. Furthermore, lack of consistent guidance on equipment for rewarming hypothermic neonates hampers recovery.

\section{Introduction}

Neonates have the highest mortality of any age group, globally (1). 2.4 million neonates died in 2019; one neonate died every 13 seconds, $80 \%$ in sub-Saharan Africa and South Asia (2) and $99 \%$ of deaths occurred in low-income and middle-income countries $(2,3)$. One in three neonatal deaths occurred within six hours of birth, $46 \%$ within 24 hours (4) and $78 \%$ within the first week (5).

Hypothermia has been widely regarded as a major contributory factor to neonatal mortality and morbidity in low-resource settings (68). The World Health Organization (WHO) defines hypothermia as a condition in which the body temperature drops below $36.5^{\circ} \mathrm{C}(6,7)$. Numerous studies have proved that hypothermia is associated with prematurity $(9-17)$, infection $(15,18-20)$ and asphyxia $(15,16$, 21-23), which are considered the three major causes of neonatal mortality (3). Already in the early 1900s, it was recognised that small neonates could not maintain their body temperature in the absence of an appropriate thermal environment (6). In the mid-1900s, Silverman, Fertig and Berger (24) and Day et al (25) demonstrated improved survival with use of warming devices to provide adequate, stable thermal care. Much later in 1993, WHO published 'Thermal Protection of the Newborn: a practical guide' to enable healthcare personnel at all levels to be adequately informed about neonatal thermal care (6).

The WHO thermal care guidelines recommend a set of 10 co-dependent steps, called the 'warm chain', to minimise heat loss in neonates and prevent hypothermia at birth and immediately thereafter $(6,7)$. The warm chain aims effective thermal care and consists of a warm delivery room, immediate drying, skin-to-skin care, early initiation of breastfeeding, delayed bathing and weighing, appropriate clothing/bedding especially the use of a hat, keeping mother and neonate together, warm transportation, warm resuscitation, as well as training and awareness raising. In addition to the warm chain, the guideline recommends other practices to prevent hypothermia after delivery, such as recognition of high-risk neonates prior to birth i.e., preterm neonates as well as measuring the temperature of these neonates at regular and frequent intervals. 
In terms of rewarming hypothermic neonates, the WHO thermal care guidelines recommend the use of external heat sources such as radiant warmers, heated mattresses and closed incubators and, if no equipment is available, skin-to-skin care for those who are clinically stable $(6,7)$. For clinically unstable hypothermic neonates, rewarming and maintaining normal temperature remains a challenge when no equipment is available (26).

Despite the recognition of the importance of thermal care for neonates a century ago and the established WHO guidelines, a high prevalence of neonatal hypothermia is still regularly reported $(14,17)$. A systematic review of the literature on prevalence studies of neonatal hypothermia in low-resource settings detailed that the prevalence in hospitals ranged from $8 \%$ within 12 hours of birth in Guinea-Bissau (defined as $<34.5^{\circ} \mathrm{C}$ ) to $85 \%$ on admission in Zimbabwe (defined as $<36.0^{\circ} \mathrm{C}$ ); and prevalence in communities from $11 \%$ within 24 hours of birth in India (defined as $<35.6^{\circ} \mathrm{C}$ ) to $92 \%$ during the first month in Nepal (defined as $<36.5^{\circ} \mathrm{C}$ ) $(14)$. Aside from the huge discrepancies in the rates of reported hypothermia, this systematic review also highlights the difficulties in comparing data due to inconsistent definitions of hypothermia. While the WHO thermal care guidelines are well recognised with over 200 citations, 21 in 2021 alone (27), they have been revised only once in 1997 and up-to-date guidance, including internationally recognised definitions of hypothermia, is lacking $(14,28)$.

Hypothermia is avoidable in the majority of neonates because it is not a complication of prematurity itself but more the result of inadequate thermal care (29-31). The high prevalence of potentially preventable hypothermia today urges an investigation into the reasons why neonates still become hypothermic despite apparent awareness of the problem and the established WHO thermal care guidelines. The objective of this study was to explore the current gaps in knowledge and practice in neonatal thermal care among healthcare workers in low-resource settings.

\section{Methods}

An expert survey was conducted to gain informed opinions on neonatal thermal care (32). In order to ensure the process was not too repetitive and time-consuming for the participants (33), we conducted a 2-round, web-based survey. We used a purposive and snowball sampling approach to invite experts in neonatal care who were physicians, nurses or midwives in neonatal care at all levels of institutions in low-resource settings globally through personal e-mail invitation. We also invited some neonatal care experts from highresource settings with extensive clinical experience in low-resource contexts to generate a more diverse range of opinions. As there is no agreed sample size for an expert survey (34), we aimed for a sample size of 30 , which was realistic to achieve with participants who were mainly from low-resource settings or had worked there. The same experts were invited for the first- and second-round surveys. Experts, who were not invited for the first-round, were additionally invited for the second-round to achieve the planned sample size.

The survey asked multiple-choice questions supplemented by open-ended questions to capture new insights and information on neonatal thermal care. The questionnaire for the first-round survey was developed using themes of neonatal thermal care extracted from existing neonatal care guidelines, including the WHO thermal care guidelines $(6,7,35-38)$. The questionnaire for the secondround survey was established based on themes which emerged from the results of the first-round survey. We provide the questionnaires for both rounds of the survey in the supplementary material. Piloting of the questionnaires was undertaken with collaborating healthcare workers. The survey was developed in English, translated into French and administered via Google Forms. Each of the two surveys took approximately 10 to 15 minutes to complete. Two weeks were given to complete the survey. The data from the survey was collected between February and June 2021. Results of the survey were analysed using Microsoft Excel version 2018 (Redmond, WA, USA). Data were collated and summarized using descriptive measures.

\section{Results}

We first present the characteristics of participants and then describe four domains of acknowledgement and the perceived practice in neonatal thermal care in low-setting resources that consist of (1) monitoring of thermal balance, (2) prevention of hypothermia, (3) management of hypothermic neonates and (4) perception and utilization of warming devices.

Characteristics of participants

As specified in Table 1, a total of 55 neonatal care professionals participated in the first-round survey: 25 medical doctors ( $45 \%), 16$ nurses (29\%), 11 midwives (20\%) and 3 clinical health officers/medical assistants (5\%); 22 participants (40\%) were from South and Southeast Asia, 21 (38\%) from Sub-Saharan Africa, three (5\%) from Middle East and 9 (17\%) from other countries. In the second-round, 33 professionals participated: 21 medical doctors (64\%), two nurses (6\%), eight midwives (24\%) and two other professionals (6\%); 18 
(55\%) of them were from Sub-Saharan Africa, six (18\%) from South and Southeast Asia and nine (27\%) from other countries. A total of 18 participated in both the first and second rounds.

Table 1

Characteristics of participants.

\begin{tabular}{|c|c|c|}
\hline Characteristics & $\begin{array}{l}\text { 1st round } \\
\mathrm{n}(\%)\end{array}$ & $\begin{array}{l}\text { 2nd round } \\
\mathrm{n}(\%)\end{array}$ \\
\hline Profession & $25(45 \%)$ & $21(64 \%)$ \\
\hline Medical doctor & $16(29 \%)$ & $2(6 \%)$ \\
\hline Nurse & $11(20 \%)$ & $8(24 \%)$ \\
\hline Midwife & $3(5 \%)$ & 0 \\
\hline $\begin{array}{l}\text { Clinical health officer/Medical assistant } \\
\text { Other }\end{array}$ & 0 & $2(6 \%)$ \\
\hline Work experience (years) & $17(31 \%)$ & $8(24 \%)$ \\
\hline$<5$ & $11(20 \%)$ & $8(24 \%)$ \\
\hline $5-9$ & $20(36 \%)$ & $8(24 \%)$ \\
\hline $\begin{array}{l}10-20 \\
>20\end{array}$ & $7(13 \%)$ & $9(27 \%)$ \\
\hline Place of work & $31(56 \%)$ & $14(42 \%)$ \\
\hline Neonatal Intensive Care Unit & $10(18 \%)$ & $7(21 \%)$ \\
\hline Delivery room & $8(14 \%)$ & $9(27 \%)$ \\
\hline Paediatric ward/intensive care unit that admits neonates & $3(5 \%)$ & $1(3 \%)$ \\
\hline $\begin{array}{l}\text { Outpatient department/Emergency room } \\
\text { Other }\end{array}$ & $3(6 \%)$ & $2(6 \%)$ \\
\hline Country of work & $22(40 \%)$ & $6(18 \%)$ \\
\hline South and Southeast Asia*1 & $21(38 \%)$ & $18(55 \%)$ \\
\hline Sub-Saharan Africa*2 & $3(5 \%)$ & 0 \\
\hline Middle East ${ }^{\star 3}$ & $9(17 \%)$ & $9(27 \%)$ \\
\hline Other*4 & & \\
\hline
\end{tabular}

*1 Afghanistan, Bangladesh, India, Indonesia, Nepal, Philippines, Thailand.

*2 Burkina Faso, Cameroon, Ethiopia, Gabon, Ivory Coast, Malawi, Nigeria, Senegal, Somalia, Uganda.

*3 Iraq, Lebanon, Yemen.

*4 Based in Japan, Switzerland and USA with extensive clinical experience in low-resource settings.

\section{Note}

Percentages may not total 100 due to rounding.

Monitoring of thermal balance 
Temperature monitoring was generally considered adequately performed by participants. They answered that the quality of temperature monitoring at their institutions was: excellent ( $33 \%$; $=18 / 55)$; good $(42 \% ; n=23 / 55)$; average $(24 \%$; $n=13 / 55)$; and poor $(2 \% ; n=1 / 55)$. Most participants also reported that hypothermia was detected early within one hour $(76 \% ; n=41 / 54)$.

Only $56 \%(n=30 / 54)$ of the participants agreed on the usefulness of checking the peripheral temperature by touching the skin of the feet or hand. As an indicator for cold stress (defined within the questionnaire as a condition in which neonates are below optimum environmental temperature and using more oxygen and energy while still maintaining normal body temperature (39)), the usefulness of the core temperature (defined within the questionnaire as rectal or axillary temperature $(6,7)$ ) was valued higher than that of the peripheral temperature (defined within the questionnaire as foot or hand temperature (40)) with $70 \%(n=23 / 33)$ and $58 \%(n=19 / 33)$ of agreement, respectively. Seven participants (five medical doctors and two midwives) commented that the peripheral temperature was an early indicator of cold stress before hypothermia would occur. Two other participants (one medical doctor and one midwife) also stated that cold extremities were a sign of vasoconstriction due to cold stress. Conversely, six participants (three medical doctors, two midwives and one other) commented that peripheral temperatures were inaccurate, thus, rectal or axillary temperature was needed to be checked for confirmation. The majority of the participants $(85 \% ; n=28 / 33)$ agreed on the nurses' and midwives' capability to detect $\mathrm{cool} /$ cold peripheral temperatures, whereas fewer participants $(61 \% ; n=20 / 33)$ agreed on the mothers' and family members' capability to do so.

\section{Prevention of hypothermia}

Table 2 summarizes reported acknowledgements on the importance of the WHO 10 steps of the warm chain and their reported practice. Almost all participants agreed on the importance of all the 10 steps of the warm chain. They reported that these steps were performed relatively well within their institution. Very few answered that any of these measures were practiced either poorly or extremely poorly. However, as shown in Table 2, the positive responses (strongly agree or agree) regarding the importance of knowledge exceeded those of the perceived quality of practice (excellent or good), especially of skin-to-skin care $(\Delta \%=25 \%)$, awareness $(\Delta \%=20 \%)$, delayed bathing and weighing at birth $(\Delta \%=18 \%)$ and warm transportation $(\Delta \%=17 \%)$. Without exception all steps scored lower for practice than knowledge.

Table 2

Acknowledgement and quality of practice of the WHO 10 steps of the warm chain $(n=49-55)$.

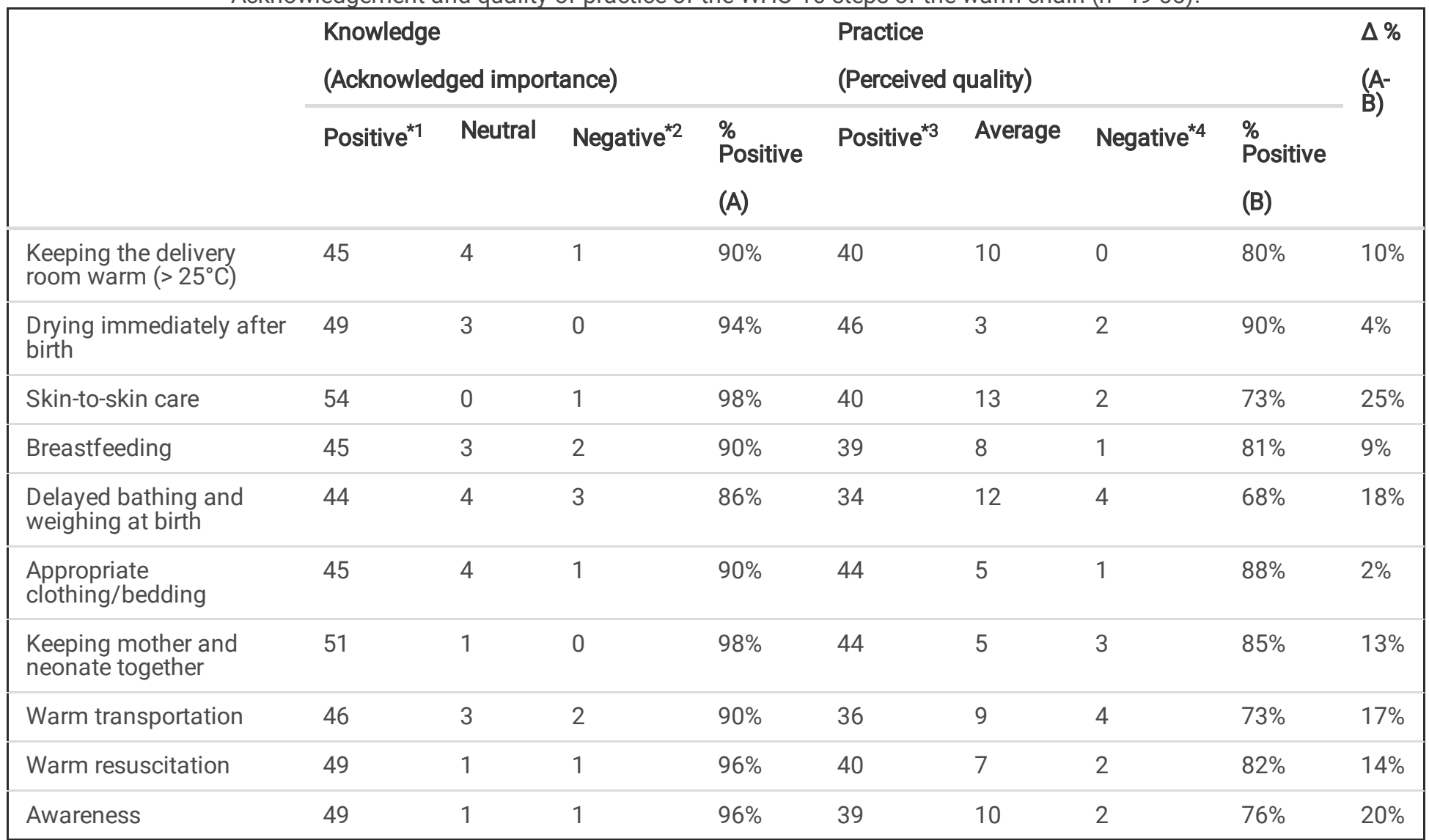


${ }^{*}$ Combining answers reporting strongly agree or agree.

${ }^{* 2}$ Combining answers reporting strongly disagree or disagree.

${ }^{* 3}$ Combining answers reporting excellent or good.

${ }^{*} 4$ Combining answers reporting extremely poor or poor.

Management of hypothermic neonates

Regarding acknowledgement of the main management strategies of hypothermic neonates and their reported quality of practice (Table 3), more than $92 \%$ of the participants agreed on the importance of keeping the room temperature warm (> $\left.25^{\circ} \mathrm{C}\right)$, skin-to-skin care, breast feeding, appropriate clothing and bedding, radiant warmer and treating underlying causes. However, only 66-76\% of them agreed on the importance of plastic wraps/bags, silver swaddlers, heated mattresses and closed incubators. These methods were either unavailable or not used in many of the participants' institutions (22-35\%). In addition, more participants reported poor or extremely poor practice of these devices compared to the rest of the methods that had higher agreement on their importance.

Table 3

Acknowledgement and quality of practice of the main management strategies to treat hypothermia $(n=50-55)$.

\begin{tabular}{|c|c|c|c|c|c|c|c|c|c|}
\hline & \multicolumn{4}{|c|}{$\begin{array}{l}\text { Knowledge } \\
\text { (Acknowledged importance) }\end{array}$} & \multicolumn{4}{|c|}{$\begin{array}{l}\text { Practice } \\
\text { (Perceived quality) }\end{array}$} & \multirow{2}{*}{$\begin{array}{l}\text { Unavailable/ } \\
\text { not used } \\
(\%)\end{array}$} \\
\hline & Positive ${ }^{\star 1}$ & Neutral & Negative ${ }^{\star 2}$ & $\begin{array}{l}\% \\
\text { Positive }\end{array}$ & Positive $^{\star 3}$ & Neutral & Negative $^{* 4}$ & $\begin{array}{l}\% \\
\text { Positive }\end{array}$ & \\
\hline $\begin{array}{l}\text { Keeping the } \\
\text { room warm }(> \\
\left.25^{\circ} \mathrm{C}\right)\end{array}$ & 48 & 3 & 0 & $94 \%$ & 40 & 9 & 3 & $77 \%$ & 0 \\
\hline Plastic wrap/bag & 34 & 13 & 4 & $67 \%$ & 25 & 6 & 5 & $69 \%$ & $\begin{array}{l}14 \\
(28)\end{array}$ \\
\hline Skin-to-skin care & 53 & 0 & 0 & $100 \%$ & 45 & 7 & 3 & $82 \%$ & 0 \\
\hline Breastfeeding & 46 & 3 & 1 & $92 \%$ & 42 & 7 & 2 & $82 \%$ & 0 \\
\hline Silver swaddler & 36 & 9 & 6 & $71 \%$ & 27 & 4 & 5 & $75 \%$ & $\begin{array}{l}16 \\
(31)\end{array}$ \\
\hline $\begin{array}{l}\text { Appropriate } \\
\text { clothing/bedding }\end{array}$ & 50 & 3 & 0 & $94 \%$ & 48 & 5 & 1 & $89 \%$ & 0 \\
\hline Radiant warmer & 47 & 3 & 1 & $92 \%$ & 42 & 5 & 3 & $84 \%$ & $\begin{array}{l}2 \\
(4)\end{array}$ \\
\hline Heated mattress & 33 & 9 & 8 & $66 \%$ & 20 & 5 & 8 & $61 \%$ & $\begin{array}{l}18 \\
(35)\end{array}$ \\
\hline Closed incubator & 38 & 11 & 1 & $76 \%$ & 32 & 5 & 3 & $80 \%$ & $\begin{array}{l}11 \\
(22)\end{array}$ \\
\hline $\begin{array}{l}\text { Treating } \\
\text { underlying cause }\end{array}$ & 47 & 1 & 2 & $94 \%$ & 45 & 3 & 2 & $90 \%$ & 0 \\
\hline
\end{tabular}

${ }^{* 1}$ Combining answers reporting strongly agree or agree.

${ }^{* 2}$ Combining answers reporting strongly disagree or disagree.

${ }^{\star 3}$ Combining answers reporting excellent or good. 
${ }^{*} 4$ Combining answers reporting extremely poor or poor.

Perception and utilization of warming devices

Radiant warmers, heated mattresses and closed incubators were assessed for 9 indicators (Table 4). Only the following three assessments out of the 27 combinations had more than $84 \%$ agreement: closed incubators (97\%; $n=31 / 32)$ and radiant warmers (91\%; $n=29 / 32$ ) were considered either effective or highly effective; the acquisition costs of closed incubators were considered either expensive or very expensive $(84 \% ; n=27 / 32)$. Fewer participants $(67 \% ; n=22 / 33)$ agreed on the effectiveness of heated mattresses, contrarily. 
Table 4

Summary of acknowledged costs, risk, effectiveness and usability of warming devices $(n=31-33)$.

\begin{tabular}{|c|c|c|}
\hline Effectiveness (it works) & $\%$ Very effective/effective & $\%$ Very ineffective/ineffective \\
\hline Radiant warmer & $91 \%$ & $3 \%$ \\
\hline Heated mattress & $67 \%$ & $3 \%$ \\
\hline Closed incubator & $97 \%$ & $3 \%$ \\
\hline Cost to purchase & $\%$ Very cheap/cheap & $\%$ Very expensive/expensive \\
\hline Radiant warmer & $19 \%$ & $61 \%$ \\
\hline Heated mattress & $34 \%$ & $28 \%$ \\
\hline Closed incubator & $3 \%$ & $84 \%$ \\
\hline Cost to run/maintain & $\%$ Very cheap/cheap & $\%$ Very expensive/expensive \\
\hline Radiant warmer & $23 \%$ & $39 \%$ \\
\hline Heated mattress & $41 \%$ & $16 \%$ \\
\hline Closed incubator & $9 \%$ & $69 \%$ \\
\hline Cost to discard & $\%$ Very cheap/cheap & $\%$ Very expensive/expensive \\
\hline Radiant warmer & $16 \%$ & $42 \%$ \\
\hline Heated mattress & $41 \%$ & $25 \%$ \\
\hline Closed incubator & $9 \%$ & $59 \%$ \\
\hline Electricity requirement & $\%$ Very low/low & $\%$ Very high/high \\
\hline Radiant warmer & $16 \%$ & $66 \%$ \\
\hline Heated mattress & $39 \%$ & $27 \%$ \\
\hline Closed incubator & $12 \%$ & $73 \%$ \\
\hline Risk of over heating & $\%$ Very low/low & $\%$ Very high/high \\
\hline Radiant warmer & $25 \%$ & $22 \%$ \\
\hline Heated mattress & $33 \%$ & $21 \%$ \\
\hline Closed incubator & $30 \%$ & $27 \%$ \\
\hline Risk of infection & $\%$ Very low/low & $\%$ Very high/high \\
\hline Radiant warmer & $53 \%$ & $9 \%$ \\
\hline Heated mattress & $36 \%$ & $18 \%$ \\
\hline Closed incubator & $18 \%$ & $48 \%$ \\
\hline Ease of use & \% Very easy/easy & $\%$ Very difficult/difficult \\
\hline Radiant warmer & $66 \%$ & $9 \%$ \\
\hline Heated mattress & $78 \%$ & $6 \%$ \\
\hline Closed incubator & $27 \%$ & $45 \%$ \\
\hline Ease of clean & \% Very easy/easy & $\%$ Very difficult/difficult \\
\hline Radiant warmer & $59 \%$ & $6 \%$ \\
\hline Heated mattress & $61 \%$ & $9 \%$ \\
\hline
\end{tabular}




\begin{tabular}{|lll|}
\hline Effectiveness (it works) & \% Very effective/effective & $\%$ Very ineffective/ineffective \\
\hline Closed incubator & $22 \%$ & $53 \%$ \\
\hline
\end{tabular}

In terms of case management, for an example case of a late preterm neonate, with axillary temperature of $34.0^{\circ} \mathrm{C}$ and respiratory distress syndrome requiring oxygen, the priority was given by respondents to use radiant warmers $(39 \% ; n=13 / 33)$, followed by closed incubators $(24 \% ; n=8 / 33)$, skin-to-skin care $(24 \% ; n=8 / 33)$, heated mattresses $(9 \% ; n=3 / 33)$ and other methods $(3 \% ; n=1 / 33)$, considering all the equipment was available. Among eight participants who answered skin-to-skin care as their method of choice, six nevertheless answered that in real life, they would use warming devices instead. On the other hand, three participants, who preferred either radiant warmers, heated mattresses, or closed incubators in theory, would use skin-to-skin care at their institution.

When asked to choose equipment for a new hospital with 100 deliveries/month and 20 admissions to the neonatal intensive care unit/month in low-resource settings, the priority was given to a mix of equipment, consisting of two radiant warmers and five heated mattresses $(50 \% ; n=16 / 32)$, followed by five radiant warmers alone (19\%; $n=6 / 32), 10$ heated mattresses alone $(19 \% ; n=6 / 32)$ and finally two closed incubators alone $(13 \% ; n=4 / 32)$.

\section{Discussion}

In this study we aimed to explore gaps in knowledge and practice in neonatal thermal care among healthcare workers in low-resource settings, that is, in monitoring, prevention and management of neonatal hypothermia, a significant contributor to the 2.4 million annual neonatal deaths worldwide (2).

Surveys are considered good tools for revealing knowledge and opinion (32) which we believe govern much of the real-life behaviour. However, one of the limitations of our study is a low diversity in participants due to the difficulty in recruiting, thus possibly limiting generalizability of the results. In addition, due to the voluntary nature of participation in the survey, the response rate of the secondsurvey by the participants of the first-survey was low and the questionnaire lacked some answers which may weaken the validity and reliability. Providing denominators where applicable, however, may have improved them. Finally, we acknowledge that not all answers are reported, mainly free text responses, which can pose a risk of selection bias, but complete data is available on request for transparency.

Knowledge gaps in the neonatal defence processes to cold stress

As the most prominent finding, we identified lacking knowledge on the importance of peripheral temperature as an early indicator of cold stress before hypothermia occurs. Unfortunately, the definition of cold stress is often confusingly reported in the literature merging its 'physiologic' definition (without actual hypothermia) and mild hypothermia, two metabolically very different states (40). In physiological terms, cold stress is a condition in which the environmental temperature is below the lower critical temperature to provide thermal neutrality (39). Even though the 'physiologic' definition of cold stress was clearly provided within the questionnaires, the core temperature maintained higher consideration than the peripheral temperature as an indicator for cold stress. Several comments too, demonstrated indiscriminate understanding of 'physiologic' cold stress and hypothermia.

In the neonate, cold stress triggers vasoconstriction before leading to metabolic heat production with major substrate and oxygen consumption and may limit other organ functions (41). Hypothermia occurs only when this metabolic surge is exhausted.

Vasoconstriction is most pronounced in the extremities (40) where it leads to acrocyanosis and decreased peripheral perfusion and lowers the temperature (7). Clinical detection of cold stress with a difference between core and peripheral temperature is largely used by neonatologists worldwide. Indeed, the literature reports that human temperature sensing in non-diabetic adults can detect smaller than $1^{\circ} \mathrm{C}$ differences (42), appropriate for detection of physiologic cold stress in neonates (40). Peripheral temperature checked by hand-touch, which is an inexpensive, easily available clinical monitoring tool, is therefore an early and sensitive parameter to detect cold stress whilst core temperature is still normal (43).

Unlike the 'physiologic' definition, the WHO thermal care guidelines define either axillary or rectal temperature of $36.0-36.4^{\circ} \mathrm{C}$ as cold stress or mild hypothermia (7) which may contribute to the confusion as demonstrated by the participants' answers. The WHO guidelines do mention that the neonate's temperature can be assessed by touching the skin of the feet as an alternative to the use of a thermometer (7). However, no attempt is made to discriminate between cold stress and hypothermia. We believe 'physiologic' cold stress should be considered the established disease, because the overrun metabolic capacity hampers normal postnatal adaptational 
processes long before the low temperature per se makes the neonate sick by reduced biochemical processing. When cold stress is detected, immediate intervention should re-establish a thermoneutral environment, in which neonates do not exhaust oxygen and energy for generating heat. When hypothermia is detected, an intervention should be focused on re-warming the neonate in addition to re-establishing the thermoneutral environment. Interventions for cold stress may be the same as for established hypothermia, especially when rewarming equipment is unavailable or limited. However, placing the cursor between cold stress and hypothermia, which are clearly two different metabolic conditions, differentiates prevention from treatment.

Gaps between knowledge and practice in preventing neonatal hypothermia

When WHO published the thermal care guidelines, the high prevalence of neonatal hypothermia was considered resulting more from the lack of knowledge rather than the lack of equipment (6). The results of this study showed that the importance of the WHO 10 steps of the warm chain were well acknowledged, but there was room for improvement in their practice. Deng et al (44) conducted a study to explore the factors which impact nurses' knowledge, perceptions and practice related to kangaroo care in neonatal intensive care units in China. They found that a majority of nurses acknowledged the benefits of kangaroo care, but depth of knowledge and awareness were inadequate, thus affecting their performance in supporting kangaroo care. In order to improve thermal care and decrease the high prevalence of neonatal hypothermia in low-resource settings today, it appears that, a deeper understanding of thermoregulation is necessary to succeed. We feel that an in depth understanding of the difference between cold stress and hypothermia will significantly fill this gap between knowledge and practice. However, knowledge alone does not assure practice. Deng et al (44) also reported that the nurses perceived increased workloads with significant time consumption of kangaroo care implementation as a disadvantage. A proper facility setup and sufficient human resources are necessary for healthcare workers to practice their knowledge of thermal care (45).

Lack of guidance on equipment to rewarm hypothermic neonates

Unlike preventive measures, opinions on rewarming methods largely differed among participants. We assessed three common warming devices, radiant warmers, heated mattresses and closed incubators, based on 9 criteria. Some preferred radiant warmers, others closed incubators and many were uncertain about the effectiveness of heated mattresses. Thus, skin-to-skin care was considered preferable to heated mattresses for rewarming even in clinically unstable neonates.

Much of these choices may directly result from the availability of these technical devices in the participants' working environment as well as the practical experience, as suggested by the difference between theoretical and practical preferences. This dichotomy warrants very careful implementation of essential technical devices, in order to avoid the use of complex and costly equipment and interventions where lower technology may be sufficient, especially when this strategy limits skin-to-skin care.

Plastic wrap/bags, silver swaddlers, heated mattresses and closed incubators were either unavailable or not used in many of the participants' institutions. A lack of published evidence on their effectiveness in low-resource settings and absent of WHO recommendations may explain some of these findings $(28,46)$. Most participants agreed that closed incubators were expensive to purchase even though these were considered to be effective which may explain low availability of closed incubators in their institutions. WHO warns that closed incubators may be more of a hazard than a benefit to the neonate and should only be used in hospitals where skilled personnel is capable of running, maintaining and repairing them and where a reliable electricity supply is warranted $(6,7)$. Based on the responses from our questionnaire, the reasons why heated mattresses remain largely unavailable or unused in many institutions appear to be the perceived uncertainty of its effectiveness. Although there is not a one-size-fits-all approach in rewarming hypothermic neonates, a pragmatic guidance for a balance between effectiveness and affordability of equipment in healthcare facilities in low-resource settings is necessary in order to avoid the use of hazardous and costly devices where low-cost methods could be similarly effective.

\section{Conclusions And Recommendations}

This study identified some bottlenecks in knowledge and practice in neonatal thermal care. First of all, an inadequate understanding of cold stress underestimates the potential benefit of peripheral temperatures checked by hand-touch for monitoring deviations from the thermoneutral zone before hypothermia occurs, and leads to missed opportunities of timely intervention to prevent hypothermia. In addition, a lack of guidance on equipment was identified in order to rewarm hypothermic neonates. These results stress the importance of knowledge but also the better implementation based on consistent context-adaptable guidance which needs to include the choice of effective and affordable warming devices without replacing skin-to-skin care. 
Through its 10 steps of the warm chain, the WHO guidelines appear to effectively transmit guidance for prevention of hypothermia, although their practical application remains more limited. However, the current guidelines fail in transmitting critical guidance required to differentiate between cold stress and hypothermia and in providing advice on the choice of low-cost technical equipment for efficient rewarming. We therefore urge for an update of the WHO guidelines including an improved definition of cold stress and hypothermia and a technical toolkit for prevention and treatment of neonatal hypothermia.

\section{Abbreviations}

WHO

World Health Organization

\section{Declarations}

Ethics approval and consent to participate: The study was considered as falling outside of the scope of the Swiss legislation regulating research on human subjects, so that the requested Swiss ethics committee approval was waived. The survey was completed anonymously and voluntarily by participants, thus, considered as informed consent.

Consent for publication: Not applicable.

Availability of data and materials: All relevant data are presented in this published article and its supplementary information files. Additional data could be available upon request to the corresponding author.

Competing interests: The authors declare that they have no competing interests.

Funding: This research received no specific grant from any funding agency in the public, commercial, or non-profit sectors.

Authors' contributions: MK and REP conceptualised and designed the study. MK was a major contributor in carrying out the implementation. MK, VJ, FRM, GBP and REP contributed to data analyses and results write up, drafting the manuscript and finalization. All authors read and approved the final manuscript.

Acknowledgements: The authors thank the following individuals for their contribution: Dr. Ahmed llyas Sh, Ms. Elizabeth Anyango Walumbe, Dr. François Niada, Dr. Kuissi Kamgaing Eliane, Mr. Mathiang Majok Deng, Dr. Nadia Lafferty, Dr. Sayori Kobayashi, Dr. Sweet C Alipon and Mr. Serge Dzeukou. We also thank all anonymous contributors to the questionnaire.

\section{References}

1. UNICEF. Neonatal mortality. Available from: https://data.unicef.org/topic/child-survival/neonatal-mortality/ [Accessed $24^{\text {th }}$ September 2021].

2. World Bank. Number of neonatal deaths. Available from: https://data.worldbank.org/indicator/SH.DTH.NMRT? end=2019\&name_desc=false\&start=1960\&view=chart [Accessed $24^{\text {th }}$ September 2021].

3. Lawn JE, Cousens S, Zupan J. 4 Million neonatal deaths: When? Where? Why?. Lancet. 2005;365: 891-900. Available from: https://doi.org/10.1016/S0140-6736(05)71048-5.

4. Baqui AH, Mitra DK, Begum N, Hurt L, Soremekun S, Edmond K, et al. Neonatal mortality within 24 hours of birth in six low- and lower-middle-income countries. Bull World Health Organ. 2016;94(10): 752-758B. Available from: https://doi.org/10.2471/BLT.15.160945.

5. Sankar MJ, Natarajan CK, Das RR, Agarwal R, Chandrasekaran A, Paul VK. When do newborns die? A systematic review of timing of overall and cause-specific neonatal deaths in developing countries. Journal of Perinatology. 2016;36(Suppl 1): S1-S11. Available from: https://doi.org/10.1038/jp.2016.27.

6. World Health Orgnatization. Thermal protection of the newborn: a practical guide. Available from: https://apps.who.int/iris/bitstream/handle/10665/60042/WHO_FHE_MSM_93.2.pdf? sequence=1\&isAllowed=y [Accessed 24 ${ }^{\text {th }}$ September 2021]. 
7. World Health Organization. Thermal protection of the newborn: a practical guide. Available from: https://apps.who.int/iris/bitstream/handle/10665/63986/WHO_RHT_MSM_97.2.pdf?sequence=1\&isAllowed=y [Accessed 24 ${ }^{\text {th }}$ September 2021].

8. Sodemann M, Nielsen J, Veirum J, Jakobsen MS, Biai S, Aaby P. Hypothermia of newborns is associated with excess mortality in the first 2 months of life in Guinea-Bissau, West Africa. Trop Med Int Heal. 2008;13(8): 980-6. Available from: https://doi.org/10.1111/j.1365-3156.2008.02113.x 9.

9. Zayeri F, Kazemnejad A, Ganjali M, Babaei G, Khanafshar N, Nayeri F. Hypothermia in Iranian newborns. Incidence, risk factors and related complications. Saudi Med J. 2005;26(9): 1367-71. Available from: https://pubmed.ncbi.nlm.nih.gov/16155649/ [Accessed 24th September 2021].

10. Zayeri F, Kazemnejad A, Ganjali M, Babaei G, Nayeri F. Incidence and risk factors of neonatal hypothermia at referral hospitals in Tehran, Islamic Republic of Iran. East Mediterr Heal J. 2007;13(6): 1308-18. Available from: https://doi.org/10.26719/2007.13.6.1308.

11. Ogunlesi TA, Ogunfowora OB, Adekanmbi FA, Fetuga BM, Olanrewaju DM. Point-of-admission hypothermia among high-risk Nigerian newborns. BMC Pediatr. 2008;8: 40. Available from: https://doi.org/10.1186/1471-2431-8-40.

12. Li M xia, Sun G, Neubauer H. Change in the body temperature of healthy term infant over the first 72 hours of life. $J$ Zhejiang Univ Sci. 2004;5(4): 486-93. Available from: https://doi.org/10.1631/jzus.2004.0486.

13. Mullany LC, Katz J, Khatry SK, LeClerq SC, Darmstadt GL, Tielsch JM. Neonatal hypothermia and associated risk factors among newborns of southern Nepal. BMC Med. 2010;8: 43. Available from: https://doi.org/ 10.1186/1741-7015-8-43.

14. Lunze K, Bloom DE, Jamison DT, Hamer DH. The global burden of neonatal hypothermia: Systematic review of a major challenge for newborn survival. BMC Med. 2013;11(1): 24. Available from: https://doi.org/10.1186/1741-7015-11-24.

15. Bower BD, Jones LF, Weeks MM. Cold injury in the newborn a study of 70 cases. Br Med J. 1960;1(5169): 303-9. Available from: https://doi.org/10.1136/bmj.1.5169.303.

16. Demtse AG, Pfister RE, Nigussie AK, McClure EM, Ferede YG, Tazu Bonger Z, et al. Hypothermia in Preterm Newborns: Impact on Survival. Glob Pediatr Heal. 2020;7: 2333794X20957655. Available from: https://doi.org/10.1177/2333794X20957655.

17. Muhe LM, McClure EM, Nigussie AK, Mekasha A, Worku B, Worku A, et al. Major causes of death in preterm infants in selected hospitals in Ethiopia (SIP): a prospective, cross-sectional, observational study. Lancet Glob Health. 2019;7(8): e1130-e1138. Available from: https://doi.org/10.1016/S2214-109X(19)30220-7.

18. Dagan R, Gorodischer R. Infections in Hypothermic Infants Younger Than 3 Months Old. Am J Dis Child. 1984;138(5): 483-5. Available from: https://doi.org/10.1001/archpedi.1984.02140430059015.

19. El-Radhi AS, Jawad MH, Mansor N, Ibrahim M, Jamil II. Infection in neonatal hypothermia. Arch Dis Child. 1983;58(2): 143-5. Available from: https://doi.org/10.1136/adc.58.2.143.

20. Hofer N, Müller W, Resch B. Neonates presenting with temperature symptoms: Role in the diagnosis of early onset sepsis. Pediatr Int. 2012;54(4): 486-90. Available from: https://doi.org/10.1111/j.1442-200X.2012.03570.x.

21. McCance RA. The maintenance of stability in the newly born: Part 2. Thermal balance. Arch Dis Child. 1959;34(178): 459-70. Available from: https://doi.org/10.1136/adc.34.178.459.

22. Burnard ED, Cross KW. Rectal temperature in the newborn after birth asphyxia. Br Med J. 1958;2(5106): 1197-9. Available from: https://doi.org/10.1136/bmj.2.5106.1197.

23. Jayasinghe D. Innate hypothermia after hypoxic ischaemic delivery. Neonatology. 2015;107(3): 220-3. Available from: https://doi.org/10.1159/000369119.

24. Silverman WA, Fertig JW, Berger AP. The influence of the thermal environment upon the survival of newly born premature infants. Pediatrics. 1958;22(5): 876-86. Available from: https://pediatrics.aappublications.org/content/pediatrics/22/5/876.full-text.pdf 
[Accessed $24^{\text {th }}$ September 2021].

25. Day RL, Caliguiri L, Kamenski C, Ehrlich F. BODY TEMPERATURE AND SURVIVAL OF PREMATURE INFANTS. Pediatrics. 1964;34: 171-81. Available from: https://pediatrics.aappublications.org/content/pediatrics/34/2/171.full-text.pdf [Accessed $24^{\text {th }}$ September 2021].

26. Morgan MC, Nambuya H, Waiswa P, Tann C, Elbourne D, Seeley J, et al. Kangaroo Mother Care for Clinically Unstable Neonates Weighing $\leq 2000$ gr: Is It Feasible at A Hospital in Uganda? Journal of global health. 2018;8(1): 010701. Available from: https://doi.org/10.7189/jogh.08.010701.

27. Google. Google Scholar. 2021. Available from: https://scholar.google.com/scholar?

$\mathrm{hl}=$ en\&as_sdt=0\%2C5\&q=Thermal+protection+of+the+newborn\%3A+a+practical+guide\&btnG $=$ [Accessed 24 ${ }^{\text {th }}$ September 2021].

28. McCall EM, Alderdice F, Halliday HL, Vohra S, Johnston L. Interventions to prevent hypothermia at birth in preterm and/or low birth weight infants. Cochrane Database Syst Rev. 2018 Feb 12;2(2): CD004210. Available from: https://doi.org/10.1002/14651858.CD004210.pub5.

29. Cordaro T, Gibbons Phalen A, Zukowsky K. Hypothermia and Occlusive Skin Wrap in the Low Birth Weight Premature Infant: An Evidentiary Review. Newborn Infant Nurs Rev. 2012;12(2): 78-85. Available from: doi.org/10.1053/j.nainr.2012.03.001.

30. McCall E, Alderdice F, Halliday H, Johnston L, Vohra S. Challenges of minimizing heat loss at birth: A narrative overview of evidence-based thermal care interventions. Newborn Infant Nurs Rev. 2014; 14(2): 56-63. Available from: doi.org/10.1053/j.nainr.2014.03.008.

31. Costello A, Francis V, Byrne A, Puddephatt V. State of the world's newborns: A report from Saving Newborn Lives. London: Save the Children and Women and Children First; 2001. Available from: http://files.eric.ed.gov/fulltext/ED458972.pdf [Accessed 24 ${ }^{\text {th }}$ September 2021].

32. Rindermann H, Becker D, Coyle TR. Survey of expert opinion on intelligence: Causes of international differences in cognitive ability tests. Front Psychol. 2016;7: 399. Available from: https://doi.org/10.3389/fpsyg.2016.00399.

33. Hsu C-C, Sandford BA. The Delphi technique: making sense of consensus. Pract Assessment, Res Eval. 2007;12(10): 1-8. Available from: https://doi.org/10.7275/pdz9-th90.

34. Santaguida P, Dolovich L, Oliver D, Lamarche L, Gilsing A, Griffith LE, et al. Protocol for a Delphi consensus exercise to identify a core set of criteria for selecting health related outcome measures (HROM) to be used in primary health care. BMC Fam Pract. 2018;19: 152. Available from: https://doi.org/10.1186/s12875-018-0831-5.

35. Beck D, Ganges F, Goldman S, Long P. Care of the Newborn Reference Manual. Washington, DC: Save the Children; 2004. Available from: https://www.healthynewbornnetwork.org/resource/care-of-the-newborn-reference-manual-2/ [Accessed $24^{\text {th }}$ September 2021].

36. Ministry of Health Eswatini. Neonatal Care Clinical Guidelines. 2018. Available from:

https://www.unicef.org/eswatini/reports/neonatal-care-clinical-guidelines [Accessed $24^{\text {th }}$ September 2021].

37. UNICEF, National Neonatology Forum. Toolkit for Setting Up Special Care Newborn Units, Stabilisation Units and Newborn Care Corners. 2018. Available from: https://www.healthynewbornnetwork.org/resource/toolkit-for-setting-up-special-care-newborn-unitsstabilisation-units-and-newborn-care-corners/ [Accessed $24^{\text {th }}$ September 2021].

38. World Health Organization. Managing newborn problems: a guide for doctors, nurses, and midwives. World Health Organization. 2003. Available from: https://apps.who.int/iris/handle/10665/42753. [Accessed $24^{\text {th }}$ September 2021].

39. IUPS Thermal Commission. Glossary of terms for thermal physiology. Jpn J Physiol. 200;51(2): 245-280. Available from: http://www.or.org/pdf/ThermalPhysiologyGlossary.pdf [Accessed $24^{\text {th }}$ September 2021]. 
40. Lyon AJ, Pikaar ME, Badger P, Mclntosh N. Temperature control in very low birthweight infants during first five days of life. Arch Dis Child Fetal Neonatal Ed. 1997;76(1): F47-50. Available from: https://doi.org/10.1136/fn.76.1.f47.

41. Knobel, R, Holditch-Davis, D, Schwartz, T, et al. Extremely low birth weight preterm infants lack vasomotor response in relationship to cold body temperatures at birth. J Perinatol. 2009;29: 814-821. Available from: doi.org/10.1038/jp.2009.99.

42. Bertelsmann FW, Heimans JJ, Weber EJM, Van Der Veen EA, Schoutent JA. Thermal discrimination thresholds in normal subjects and in patients with diabetic neuropathy. J Neurol Neurosurg Psychiatry. 1985;48(7):686-90. Available from: https://doi.org/10.1136/jnnp.48.7.686.

43. Israel DJ, Pozos RS. Synchronized slow-amplitude modulations in the electromyograms of shivering muscles. J App/ Physiol.1989;66(5): 2358-63. Available from: https://doi.org/10.1152/jappl.1989.66.5.2358.

44. Deng Q, Zhang Y, Li Q, Wang H, Xu X. Factors that have an impact on knowledge, attitude and practice related to kangaroo care: National survey study among neonatal nurses. J Clin Nurs. 2018;27(21-22): 4100-4111. Available from: https://doi.org/10.1111/jocn.14556.

45. Chan GJ, Labar AS, Wall S, Atun R. Kangaroo mother care: A systematic review of barriers and enablers. Bulletin of the World Health Organization. 2016;94(2): 130-141J. Available from: https://doi.org/10.2471/BLT.15.157818.

46. World Health Organization. WHO recommendations on newborn health: guidelines approved by the WHO Guidelines Review Committee. World Health Organization. 2017. Available from: https://apps.who.int/iris/handle/10665/259269. [Accessed 24th September 2021].

\section{Supplementary Files}

This is a list of supplementary files associated with this preprint. Click to download.

- Supplimentarymaterial.docx 